\title{
Erratum to: Skeletal Relapse After Mandibular Setback in Bi Max Surgery: Intraoral Vertical Ramus versus Bilateral Sagittal Split Osteotomies
}

\author{
Ra'ed M. A. Al-Delayme • Moutaz Al-Khen
}

Published online: 7 October 2014

(C) The Association of Oral and Maxillofacial Surgeons of India 2014

Erratum to: J. Maxillofac. Oral Surg. (2014)

DOI 10.1007/s12663-013-0555-y

The affiliation of the corresponding author was listed incorrectly. The correct affiliation is
R. M. A. Al-Delayme

Oral and Maxillofacial Surgery Department, Faculty of Dentistry, Dijlah University College, Baghdad, Iraq

The online version of the original article can be found under doi:10.1007/s12663-013-0555-y.

R. M. A. Al-Delayme ( $\square)$

Oral and Maxillofacial Surgery Department, Faculty of

Dentistry, Dijlah University College, Baghdad, Iraq

e-mail: raedmaxfax@yahoo.com

R. M. A. Al-Delayme

Oral and Maxillofacial Surgery Department, AL-Yarmuk

Teaching Hospital, Baghdad, Iraq

M. Al-Khen

Oral and Maxillofacial Surgery Department, Damascus Hospital,

Ministry of Health, Damascus, Syrian Arab Republic 